\title{
Multi-component Dust-ion-acoustic Solitary and Shock Waves in an Unmagnetized Dusty Plasma with Kappa Distributed Superthermal Electrons
}

\author{
O. Rahman \\ Department of Physics, Mawlana Bhashani Science and Technology University, Santosh, Tangail, Bangladesh \\ Email: armanphy203@gmail.com
}

\begin{abstract}
A theoretical investigation has been carried out for understanding the nonlinear propagation characteristics of the dust-ion-acoustic (DIA) solitary and shock waves in an unmagnetized dusty plasma containing Maxwellian negative ions, superthermally distributed electrons, positively charged stationary dust particles and cold mobile inertial positive ions. The reductive perturbation method has been employed to derive the Korteweg-de Vries (K-dV), modified Korteweg-de Vries $(\mathrm{mK}-\mathrm{dV})$ as well as Burger equations along with its solutions in order to study the basic features of such waves both numerically and analytically. It has been found that the presence of superthermal electrons significantly modifies the basic properties of such DIA solitary and shock wave structures. The present investigation can be very effective for understanding and studying various astrophysical environments (viz. Saturn magnetosphere, pulsar magnetosphere, etc).
\end{abstract}

Keywords: Dust-ion-acoustic waves, solitary waves, shock waves, superthermal electron, K-dV, $\mathrm{mK}-\mathrm{dV}$ and Burger equation.

\section{Introduction}

Nowadays the studies on the nonlinear propagation of DIA waves (e.g. DIA solitary and DIA shock waves) in dusty plasmas have received a considerable attention because of their significant importance to understand the behavior of space plasmas viz. supernovas, pulsar environments, cluster explosions and active galactic nuclei $[1,2,3,4]$. The presence of highly positively charged, massive grains of dust particles in an electron ion plasma is responsible for the appearance of new types of waves, depending on whether the dust grains are considered to be static or mobile. One type of these waves is the the DIA wave, which is nothing but the usual ion-acoustic wave modified by the presence of dust grains. In these waves, the ion mass provides the inertia of the waves and restoring force comes from the thermal pressure of electrons. The phase speed of such DIA waves is much larger than the ion thermal speed and much smaller than the electron thermal speed. Other types of waves are Dust-acoustic (DA) waves in which dust mass provides the inertia of the waves and restoring force comes from the thermal pressure of electrons as well as ions.

About 24 years ago, Shukla and Silin [5] have theoretically showed that because of the equilibrium charge neutrality condition $n_{i 0}=n_{e 0}+Z_{d} n_{d 0}$, and the strong inequality $n_{e 0} \ll n_{i 0}$ (where $n_{e 0}, n_{d 0}$, and $n_{i 0}$ are electron, dust, and ion number densities at equilibrium respectively, and $Z_{d}$ is the number of electrons residing onto the dust grain surface) a dusty plasma supports the low frequency DIA wave. The existence of such DIA waves has been found experimentally by Barkan et al. and Nakamura et al.[6,7]. The linear $[5,6,8,9]$ and nonlinear $[6,7,10,11,12,13]$ properties of DIA waves in dusty plasmas are now well understood from theoretical and experimental point of view. Mamun et al. $[14,15,16]$ have considered a dusty electronegative plasma containing Maxwellian electrons, Maxwellian negative ions, cold mobile positive ions, and negatively charged stationary dust, and have examined the possibility for the formation of IA and DIA solitary waves and double layers in a DENP. Rahman and Mamun have investigated the DIA solitary waves in dusty plasma with arbitrarily charged dust and vortex-like electron[17]. They have derived $\mathrm{mK}-\mathrm{dV}$ equations and found only positive potential due to trapped electrons. Kundu and Mamun have considered three components unmagnetized dusty plasma and studied the nonlinear propagation of DIA solitary waves with arbitrarily charged stationary dust and non-thermaly distributed electrons[18]. They have shown that the DIA waves can support solitary structures associated with both positive and negative potential due to the presence of non-thermal electrons in their dusty plasma model. 
However, a lot of theoretical observations of space plasmas $[19,20,21,22,23,24,25,26]$ are often characterized by a particle distribution function with high energy tail and they may deviate from the Maxwellian distribution. Superthermal particles may arise due to the effect of external forces acting on the natural space environment plasmas or to wave particle interaction. Plasmas with an excess of superthermal non-Maxwellian electrons are generally characterized by a long tail in the high energy region. Such space plasmas can be modeled by generalized Lorentzian or kappa distribution[19,27,28,29] rather than the Maxwellian distribution.

The isotropic three dimensional generalized Lorentzian or kappa velocity distribution function takes the form [27].

$$
F_{k}(v)=\frac{\Gamma(k+1)}{\left(\pi k \theta^{2}\right)^{3 / 2} \Gamma(k-1 / 2)}\left(1+\frac{v^{2}}{k \theta^{2}}\right)^{-(k+1)}
$$

where $\theta$ is the most probable (effective thermal) speed related to the usual thermal velocity $V_{t}=$ $\left(k_{B} T / m\right)^{1 / 2}$ by $\theta=[(2 k-3) / k] V_{t}, T$ being the characteristic kinetic temperature, i.e., the temperature of the equivalent Maxw-ellian with the same average kinetic energy [30], and $k_{B}$ is the Boltzmann constant. The parameter $k$ represents the strength of superthermality of the plasma species and it's values lies within the range $\infty>k>3 / 2$. Low values of $k$ represent a Şhard $\check{T}$ spectrum with a strong non-Maxwellian (power law-like) tail, an enhanced velocity distribution at low speeds, and a depressed distribution at intermediate speeds [27]. In the limit $k \rightarrow \infty$, the above kappa distribution function for electrons reduces to the well known Maxwell-Boltzmann distribution. The solitary waves, which are formed due to the balance between nonlinearity and dispersion, are being investigated by using a suitable set of stretched coordinates[31], which neglect the effect of dissipation. On the other hand the shock waves, which are formed due to the balance between nonlinearity and dissipation, are investigated by using a suitable set of stretched coordinates[32], which neglect the effect of dispersion. Baluku et al [33] have studied the DIA solitons in an unmagnetized dusty plasma which consist cold dust particles, adiabatic fluid ions and electrons satisfying $k$ distribution. On the other hand, the DIA shock waves in a two electron temperature dusty plasma have been investigated by Masud et al [4]. They [4] have considered an unmagnetized dusty plasma containing inertial ions, Maxwellian electrons with two distinctive temperatures and negatively charged immobile dust particles and have found the existance both positive and negative shock waves by deriving Burger equation. Alam et al [34] have studied the effect of bi-kappa distributed electrons on the nonlinear propagation DIA shock waves in a dusty supperthermal plasmas. They have investigated the effects of ion kinematic viscosity and the superthermal two temperature electrons. The roles of superthermal electrons and adiabatic heavy ions on heavy-ion-acoustic solitary and shock waves in a multi-component plasma have been studied by Shah et al [35] in 2015. However, all of the works $[4,33,34,35]$ on DIA solitary and shock waves are based on the three components (i.e., electrons, ions and dust or electrons with two distinct temperatures, ion and dust) dusty plasma but they did not consider the existence of both positive and negative ions in the same plasma system. In this paper, we investigate both the properties of solitary and shock waves separately under different conditions using the same plasma model. To the best of our knowledge, there is no investigation which has been made on DIA solitary and shock structures in multi-component dusty plasma system consisting of inertial positive ions, superthermal electrons, Maxwellians negative ions, and positively charged stationary dust particles. Therefore, in our present work, we consider a more general multi-component dusty plasma system that is an admixture of electrons, positive ions, negative ions and positively charged static dust, and examine the possibility for the formation of DIA solitary and shock structures, and analyze their basic features in such a multi-component dusty plasma system.

The manuscript is organized as follows. The basic equations governing the nonlinear propagation of DIA waves in an unmagnetized dusty plasma system are given in Sec. 2. The K-dV, mK-dV equations are derived and numerically solved in Sec. 3. The Burgers equation is also derived and numerically solved in Sec. 4. A brief discussion is finally presented in Sec. 5 .

\section{Governing Equations}

A collisionless unmagnetized four components dusty electronegative plasma (DENP) containing Maxwellian negative ions, kappa distributed electrons, cold mobile positive ions and positively charged stationary 
dust is considered. Therefore, at equilibrium, $n_{p 0}+Z_{d} n_{d 0}=n_{e 0}+n_{n 0}$, where $n_{p 0}, n_{e 0}, n_{n 0}$, and $n_{d 0}$ are, respectively, positive ion, electron, negative ion, and dust number density at equilibrium. $Z_{d}$ is the charge state of stationary dust grain. The nonlinear dynamics of the low frequency electrostatic perturbation mode in such a DENP is described by

$$
\begin{aligned}
& \frac{\partial n_{p}}{\partial t}+\frac{\partial}{\partial x}\left(n_{p} u_{p}\right)=0 \\
& \frac{\partial u_{p}}{\partial t}+u_{p} \frac{\partial u_{p}}{\partial x}=-\frac{\partial \phi}{\partial x}+\eta \frac{\partial^{2} u_{p}^{(1)}}{\partial \xi^{2}} \\
& \frac{\partial^{2} \phi}{\partial x^{2}}=\mu_{e}\left(1-\frac{\phi}{k-3 / 2}\right)^{(1 / 2-k)}+\mu_{n} e^{\alpha \phi}-n_{p}-\mu_{d}
\end{aligned}
$$

where $n_{p}\left(n_{n}\right)$ is the positive (negative) ion number density normalized by its equilibrium value $n_{p 0}\left(n_{n o}\right)$, $u_{p}$ is the positive ion fluid speed normalized by the positive ion-acoustic speed $C_{p}=\left(k_{B} T_{e} / m_{p}\right)^{1 / 2}, \phi$ is the electrostatic wave potential normalized by $k_{B} T_{e} / e, \mu_{n}=1-\mu_{e}+\mu_{d}, \mu_{d}=Z_{d} n_{d 0} / n_{p 0}, \mu_{e}=n_{e 0} / n_{p 0}$, $\alpha=T_{e} / T_{n}, T_{e}\left(T_{n}\right)$ is the electron (negative ion) temperature, $m_{p}$ is the mass of positive ion, $\eta$ is the positive ion kinematic viscosity normalized by $m_{p} n_{p 0} \omega_{p p} \lambda_{D}^{2}$ and $e$ is the magnitude of the electron charge. The time variable $t$ is normalized by the positive ion plasma period $\omega_{p p}^{-1}$ and the space variable is normalized by $\lambda_{D}$.

\section{Solitary Waves}

\subsection{Derivation of K-dV Equation}

To investigate the behavior of the small, but finite amplitude electrostatic DIA waves in the DENP under consideration, we first derive the well known Korteweg-de Vries (K-dV) equation using the reductive perturbation method[31]. In this case, we will first omit the dissipation effect by neglecting the 2 nd term on the right side of Eq. 2. Therefore, no significant dissipation effect arises in solitary structures. The $\mathrm{K}-\mathrm{dV}$ equation has been introduced by the following stretched coordinates[31,36,37,38]

$$
\left.\begin{array}{l}
\xi=\epsilon^{1 / 2}\left(x-v_{p} t\right) \\
\tau=\epsilon^{3 / 2} t
\end{array}\right\}
$$

where $\epsilon$ is a small dimensionless expansion parameter measuring the strength of nonlinearity $(0<\epsilon<1)$ and $v_{p}$ is the phase speed of this wave. We can expand the physical quantities $\left(n_{p}, u_{p}, \phi\right)$ appearing in the basic equations (1-3) in power series of $\epsilon[31,39,40,41]$. Let $M$ be any of the system variables $n_{p}, u_{p}$ and $\phi$, describing the system's state at given position and instant. We consider small deviations from the equilibrium state $M^{(0)}$-which explicitly is $n_{p}^{(0)}=1, u_{p}^{(0)}=0$ and $\phi^{(0)}=0$ by taking

$$
M=M^{(0)}+\sum_{n=1}^{\infty} \epsilon^{n} M^{(n)}
$$

Now, using Eqs. (4)-(5) into Eqs. (1)-(3) one can obtain the first order continuity equation, momentum equation and Poisson's equation which, after simplification, yield

$$
\begin{aligned}
& u_{p}^{(1)}=\frac{\psi}{v_{p}}, \quad n_{p}^{(1)}=\frac{\psi}{v_{p}^{2}} \\
& v_{p}=\sqrt{\frac{\kappa_{2}}{\kappa_{2} \alpha \mu_{n}+\kappa_{1} \mu_{e}}}
\end{aligned}
$$

where $\psi=\phi^{(1)}, \kappa_{1}=\kappa-1 / 2$ and $\kappa_{2}=\kappa-3 / 2$. Equation (7) represents the linear dispersion relation for DIA waves. In order to obtain the next higher order of $\epsilon$, we put the values of Eqs. (4)-(7) into Eqs. (1)-(3) and we get the flowing set of equations 


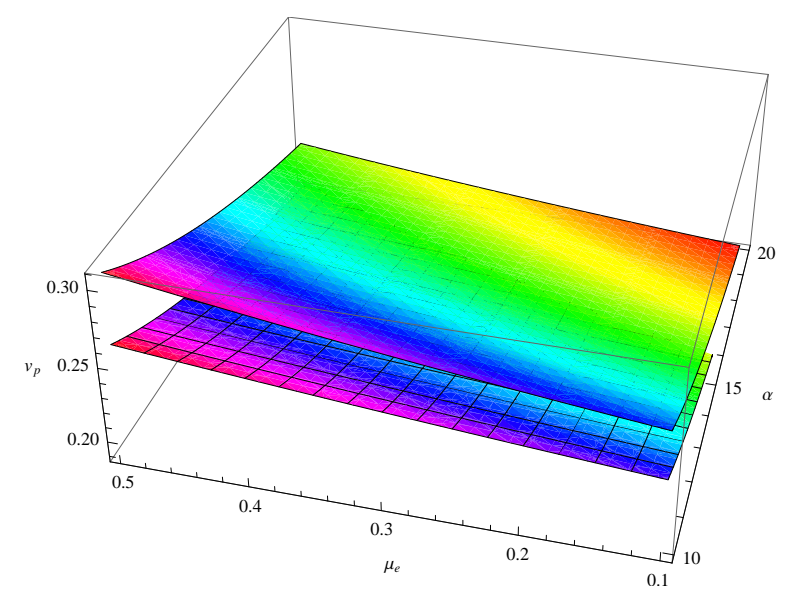

Figure 1. The variation of phase speed $\left(v_{p}\right)$ of SW with $\alpha$ and $\mu_{e}$ for $k=20$ and $\mu_{d}=0.5\left(\mu_{d}=0.9\right)$ upper surface (lower surface).

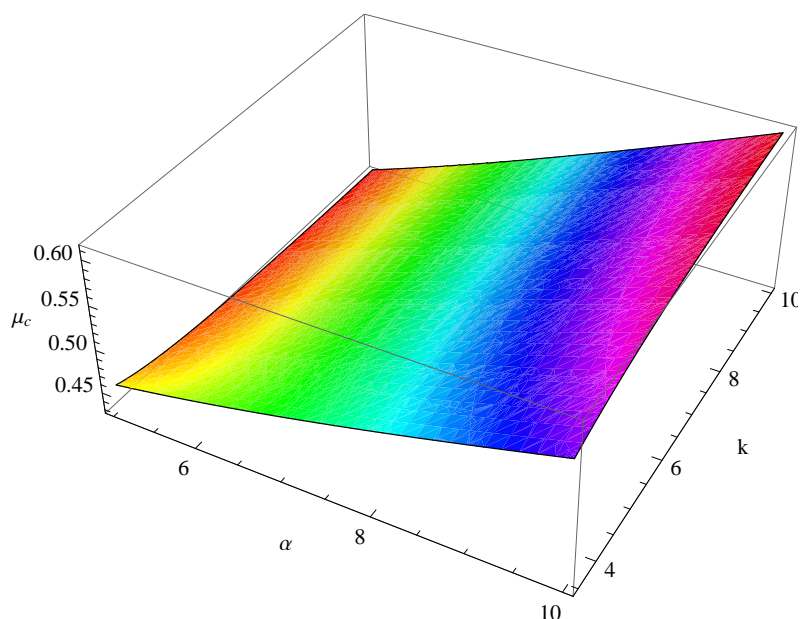

Figure 2. The $A=0$ graph which represents the variation of $\mu_{c}$ with $\alpha$ and $k$ for $\mu_{n}=0.01$. Where $\mu_{c}$ is the minimum (critical) value of $\mu_{e}$ above (below) which compressive (rarefactive) DIA solitary structures are found.

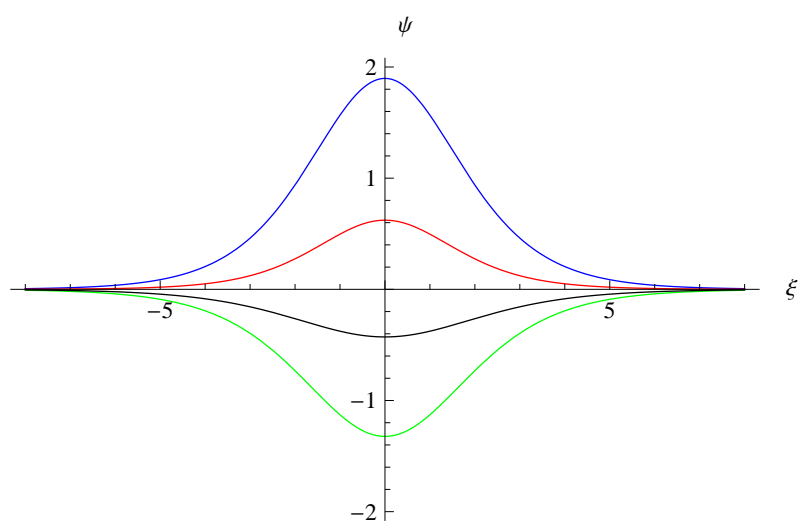

Figure 3. The variation of amplitude $\left(\psi_{m}()\right.$ of the positive and negative K-dV solitons with $\mu_{e}$ for $u_{0}=0.1$, $\mu_{n}=0.1, \alpha=10$ and $k=2$. The blue curve for $\mu_{e}=0.5$, the red curve for $\mu_{e}=0.6$, the green curve for $\mu_{e}=0.4$ and the black curve for $\mu_{e}=0.3$. 


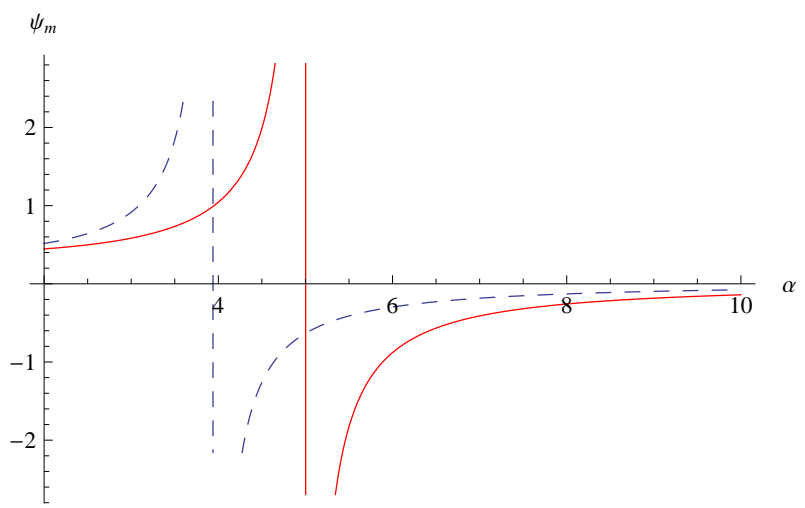

Figure 4. The variation of amplitude $\left(\psi_{m}\right)$ of solitary wave with $\alpha$ for $u_{0}=0.1, \mu_{n}=0.1$ and $\mu_{e}=0.8$. The solid curve (red) for $k=5$ and the dash curve (blue) for $k=10$.

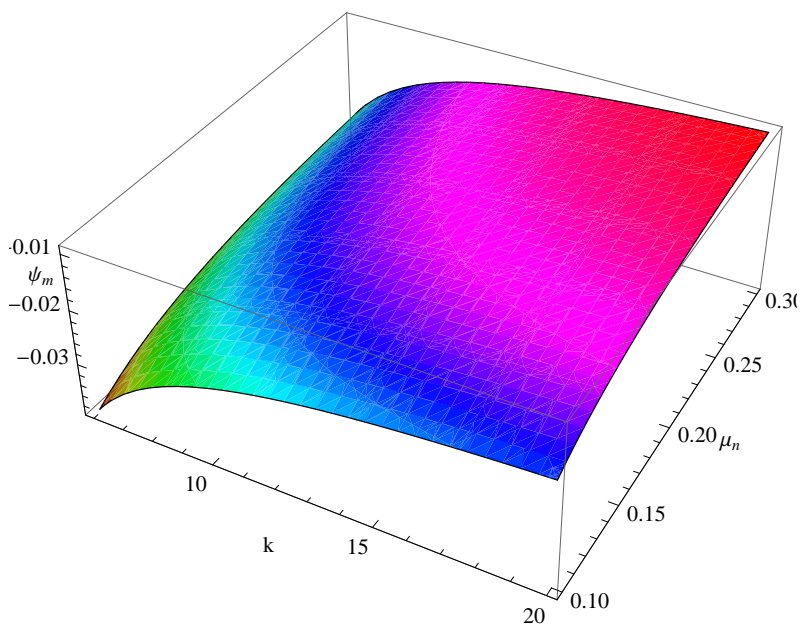

Figure 5. The variation of amplitude $\left(\psi_{m}\right)$ of solitary wave with $k$ and $\mu_{n}$ for $u_{0}=0.1, \mu_{e}=0.4$ and $\alpha=10$.

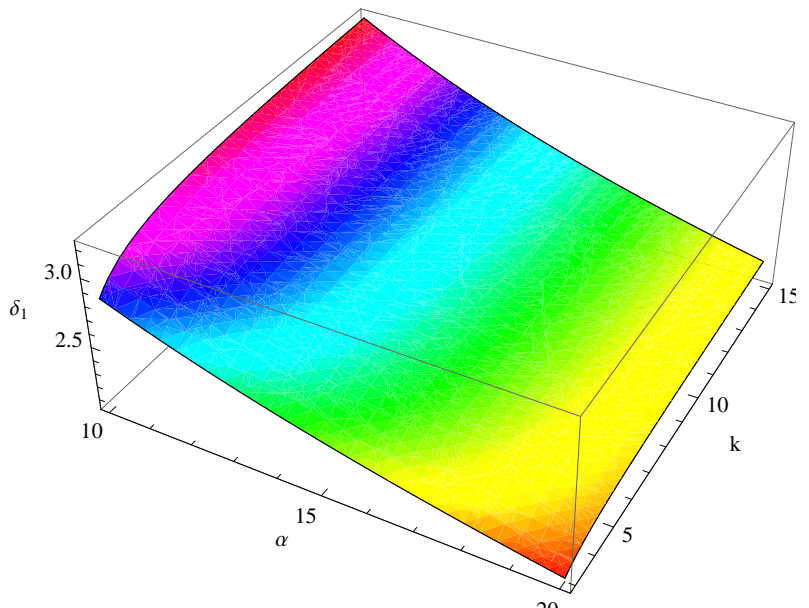

Figure 6. The variation of width $\left(\delta_{1}\right)$ of solitary wave structures with $\alpha$ and $k$ for $u_{0}=0.1, \mu_{n}=0.1$ and $\mu_{e}=0.5$. 


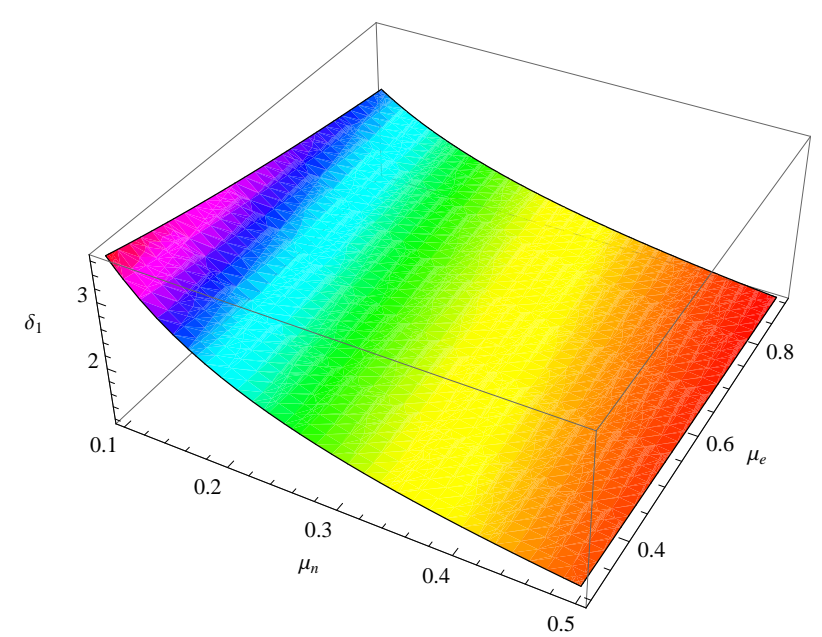

Figure 7. The variation of width $\left(\delta_{1}\right)$ of solitary wave structures with $\mu_{n}$ and $\mu_{e}$ for $u_{0}=0.1, k=20$ and $\alpha=10$.

$$
\begin{aligned}
& \frac{\partial n_{p}^{(1)}}{\partial \tau}-v_{p} \frac{\partial n_{p}^{(2)}}{\partial \xi}+\frac{\partial u_{p}^{(2)}}{\partial \xi}+\frac{\partial}{\partial \xi}\left(n_{p}^{(1)} u_{p}^{(1)}\right)=0 \\
& \frac{\partial u_{p}^{(1)}}{\partial \tau}-v_{p} \frac{\partial u_{p}^{(2)}}{\partial \xi}+u_{p}^{(1)} \frac{\partial u_{p}^{(1)}}{\partial \xi}+\frac{\partial \phi^{(2)}}{\partial \xi}=0 \\
& \frac{\partial^{2} \psi}{\partial \xi^{2}}=\frac{\phi^{(2)}}{v_{p}^{2}}+\left(\frac{\kappa_{3} \kappa_{4} \mu_{e}}{2 \kappa_{2}^{2}}+\frac{\alpha^{2} \mu_{n}}{2}\right) \psi^{2}-n_{p}^{(2)}
\end{aligned}
$$

where $\kappa_{3}=1 / 2-k, \kappa_{4}=-1 / 2-k, \kappa_{5}=\kappa+3 / 2, \kappa_{2}=k-3 / 2$. Now, solving Eqs. (8-10) with the help of Eqs. (6)-(7), we obtain a equation of the form:

$$
\frac{\partial \psi}{\partial \tau}+A \psi \frac{\partial \psi}{\partial \xi}+B \frac{\partial^{3} \psi}{\partial \xi^{3}}=0
$$

where,

$$
\begin{aligned}
& A=\frac{v_{p}^{3}}{2}\left[\frac{3}{v_{p}^{4}}-\frac{\kappa_{3} \kappa_{4} \mu_{e}}{\kappa_{2}^{2}}-\mu_{n} \alpha^{2}\right], \\
& B=\frac{v_{p}^{3}}{2} .
\end{aligned}
$$

Equation (11) is a K-dV equation, exhibiting a stronger nonlinearity, smaller width, and larger propagation velocity of the nonlinear wave.

\subsection{Solution of K-dV Equation}

The stationary solitary wave solution of this $\mathrm{K}-\mathrm{dV}$ equation can be obtained by considering a moving frame (moving with speed $\left.u_{0}\right) \zeta=\xi-u_{0} \tau$, where $\zeta$ is normalized by $\lambda_{D}$ and $u_{0}$ is normalized by $C_{p}$, and imposing the appropriate boundary conditions for localized disturbances, viz. $\psi \rightarrow 0, d \psi / d \zeta \rightarrow 0$, $d^{2} \psi / d \zeta^{2} \rightarrow 0$ at $\zeta \rightarrow \pm \infty$. Thus, one can express the stationary solitary wave solution of the K-dV equation (11) as

$$
\psi=\psi_{m} \operatorname{sech}^{2}\left[\left(\xi-u_{0} \tau\right) / \delta_{1}\right]
$$

where $\phi_{m}=3 u_{0} / A$ is the amplitude, and $\delta_{1}=\sqrt{4 B / u_{0}}$ is the width of the solitary waves. The nonlinear coefficient, A, can be either positive or negative depending on the value of $V_{p}, k, \mu_{e}, \mu_{n}$ and $\sigma$. It is clear from Eqs. (12) and (14) that the plasma system supports the DIA solitary waves with either positive 
$(A>0)$ if $\mu_{e}>0.456$ or negative potential $(A<0)$ if $\mu_{e}<0.456$ and no solitary waves exist at $A=0$. To have some numerical estimations of our results, we have numerically analyzed solitary height, width, and profile by using the general expressions for the coefficients A and B [i.e., by using Eqs. (12)-(14)]. The phase speed of DIA SWs varies with $\mu_{e}$ and $\alpha$ which is shown in figure 1. Figure 2 shows how $\mu_{c}$ varies with $\alpha$ and $k$. This represents the $\mathrm{A}=0$ surface plots, and provides us the parametric regimes above (below) which SWs associated with hump (dip) shape. The existence of both positive and negative K-dV solitons is shown in figure 3 for different values of $\mu_{e}$. The critical value of $\mu_{e}$ is 0.456 . Above this values of $\mu_{e}$ the K-dV solitons associated with only positive potential and for $\mu_{e} \leq 0.456$ the K-dV solitons associated with only negative potential. The variation of positive and negative K-dV solitons with $\alpha$ is shown in figure 4 . From this figure it has been clear that for lower values of $\alpha(\alpha<5)$ the K-dV solitons associated with positive potential and for higher values of $\alpha(\alpha>5)$ the K-dV solitons associated with negative potential. The critical value of $\alpha$ is changes with $k$. It increases with increasing the values of $k$. Figure 5 shows the variation of the potential of K-dV solitons with $k$ and $\mu_{n}$. The variations of the width $\left(\delta_{1}\right)$ of the $\mathrm{K}-\mathrm{dV}$ solitons is shown in figure 6 and 7 with some dusty plasma parameters. The width of this $\mathrm{K}-\mathrm{dV}$ solitons becomes lower with increasing the values of $\alpha, \mu_{n}$ and $\mu_{e}$. On the other hand, it becomes larger for large values of spectral index $(k)$.

\subsection{Derivation of $\mathrm{mK}-\mathrm{dV}$ Equation}

A modified $\mathrm{K}-\mathrm{dV}(\mathrm{mK}-\mathrm{dV})$ equation is obtained by taking the next higher order calculation of $\epsilon$. To analyze the nonlinear evolution near the critical parameter $\mu_{e} \simeq \mu_{c}, \mathrm{mK}$-dV equation is obtained from the third order calculation of $\epsilon$, which utilizes another set of stretched coordinates. The stretched co-ordinates $[42,43]$ for $\mathrm{mK}-\mathrm{dV}$ equation is:

$$
\left.\begin{array}{l}
\xi=\epsilon\left(x-v_{p} t\right) \\
\tau=\epsilon^{3} t
\end{array}\right\}
$$

Now, using Eq. (5) and (15) into Eqs. (1)-(3), we have found the same values of $n_{p}^{(1)}, u_{p}^{(1)}$ and $v_{p}$ as like as that of the K-dV equation. To the next higher order of $\epsilon$, we obtained a set of equations, which after using the values of $n_{p}^{(1)}, u_{p}^{(1)}$ and $v_{p}$ can be simplified as

$$
\begin{aligned}
& u_{p}^{(2)}=\frac{1}{2 v_{p}^{3}} \psi^{2}+\frac{\phi^{(2)}}{v_{p}} \\
& n_{p}^{(2)}=\frac{3}{2 v_{p}^{4}} \psi^{2}+\frac{\phi^{(2)}}{v_{p}^{2}} \\
& \rho^{(2)}=-\frac{1}{2} A\left[\psi^{(1)}\right]^{2}=0
\end{aligned}
$$

To the next higher order of $\varepsilon$, we obtain a set of equations:

$$
\begin{gathered}
\frac{\partial n_{p}^{(1)}}{\partial \tau}-v_{p} \frac{\partial n_{p}^{(3)}}{\partial \xi}+\frac{\partial u_{p}^{(3)}}{\partial \xi}+\frac{\partial}{\partial \xi}\left(n_{p}^{(2)} u_{p}^{(1)}\right)+\frac{\partial}{\partial \xi}\left(n_{p}^{(1)} u_{p}^{(2)}\right)=0 \\
\frac{\partial u_{p}^{(1)}}{\partial \tau}-v_{p} \frac{\partial u_{p}^{(3)}}{\partial \xi}+u_{p}^{(1)} \frac{\partial u_{p}^{(2)}}{\partial \xi}+u_{p}^{(2)} \frac{\partial u_{p}^{(1)}}{\partial \xi}+\frac{\partial \phi^{(3)}}{\partial \xi}=0 \\
\frac{\partial^{2} \psi}{\partial \xi^{2}}=\left[\alpha \mu_{n}+\frac{\kappa_{1} \mu_{e}}{\kappa_{2}}\right] \phi^{(3)}+\left[\frac{\kappa_{3} \kappa_{4} \kappa_{5} \mu_{e}}{6 \kappa_{2}^{3}}+\frac{\alpha^{3} \mu_{n}}{6}\right] \psi^{3}+\left[\frac{\kappa_{3} \kappa_{4} \mu_{e}}{\kappa_{2}^{2}}+\alpha^{2} \mu_{n}\right] \psi \phi^{(2)}-n_{p}^{(3)}
\end{gathered}
$$

Now, combining Eqs. (19-21), we obtain a equation of the from:

$$
\frac{\partial \psi}{\partial \tau}+\beta B \psi^{2} \frac{\partial \psi}{\partial \xi}+B \frac{\partial^{3} \psi}{\partial \xi^{3}}=0
$$

where,

$$
\beta=\frac{15}{2 v_{p}^{6}}-\frac{\kappa_{3} \kappa_{4} \kappa_{5} \mu_{e}}{2 \kappa_{2}^{2}}-\frac{\mu_{n} \alpha^{3}}{2}
$$




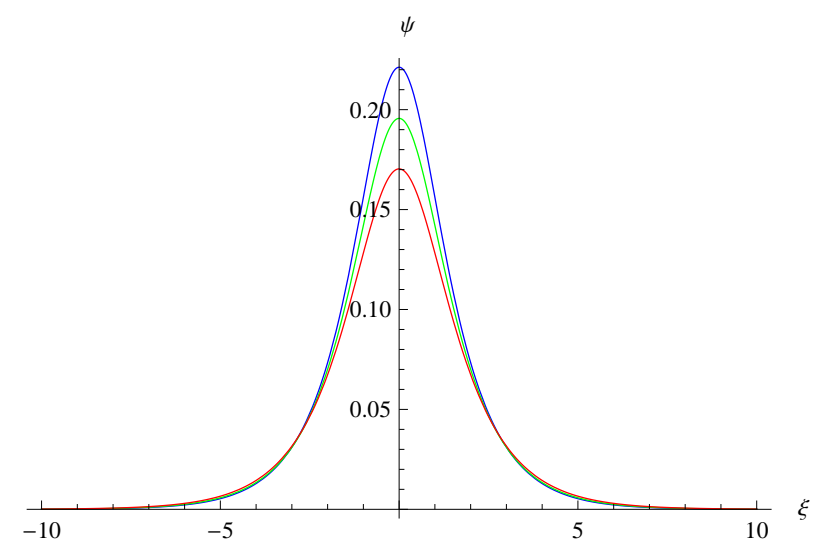

Figure 8. The variation of amplitude of the positive mK-dV solitons with $\mu_{e}$ for $u_{0}=0.1, \mu_{n}=0.3, \alpha=10$ and $k=10$. The upper curve (blue) is for $\mu_{e}=0.4$, the middle curve (green) for $\mu_{e}=0.6$ and the lower curve (red) for $\mu_{e}=0.9$.

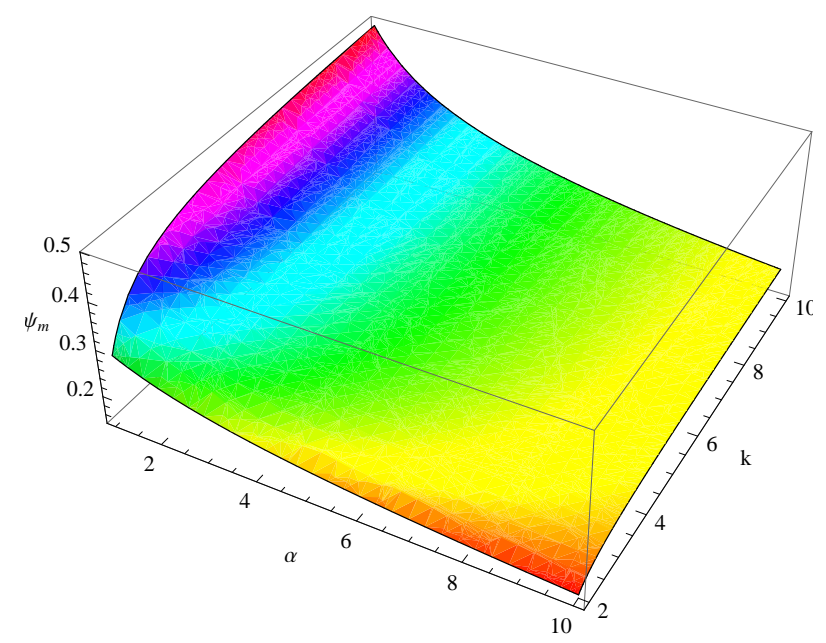

Figure 9. The variation of amplitude of the positive mK-dV solitons with $\alpha$ and $k$ for $u_{0}=0.1, \mu_{n}=0.3$ and $\mu_{e}=0.5$.

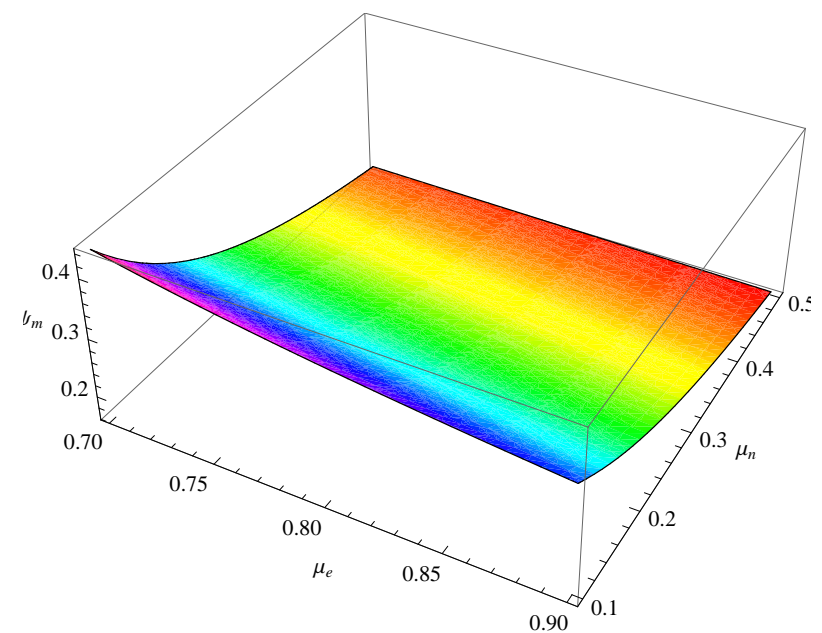

Figure 10. The variation of amplitude of the positive mK-dV solitons with $\mu_{e}$ and $\mu_{n}$ for $u_{0}=0.1, \alpha=5$ and $k=10$. 


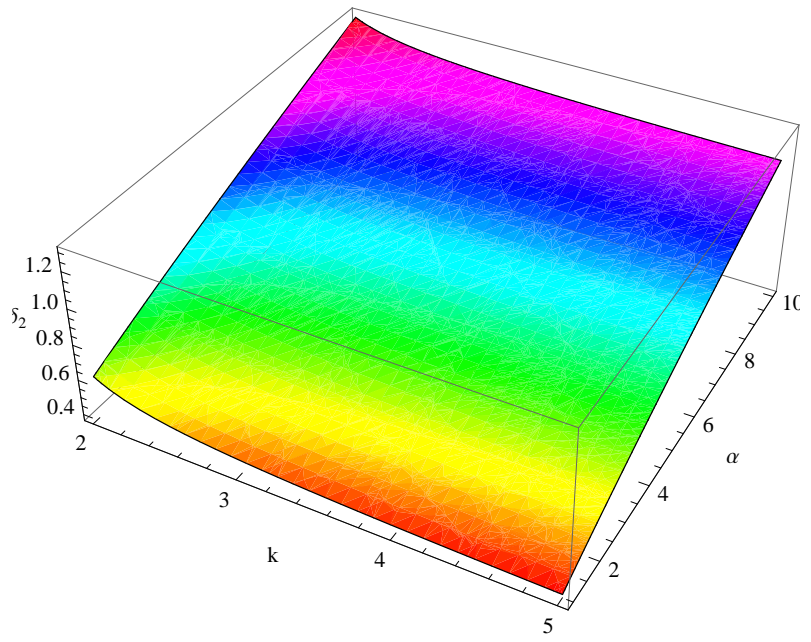

Figure 11. The variation of width of the positive mK-dV solitons with $k$ and $\alpha$ for $u_{0}=0.1, \mu_{n}=0.3$ and $\mu_{e}=0.4$.

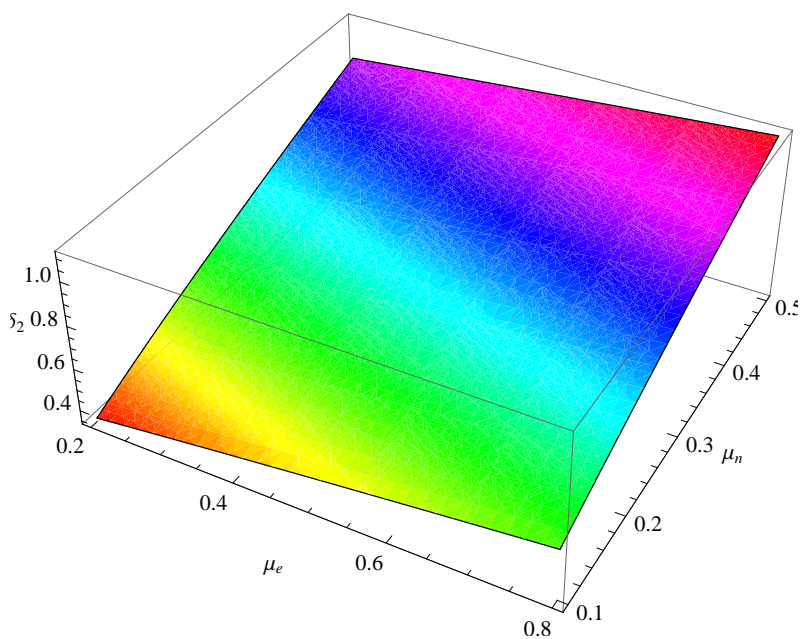

Figure 12. The variation of width of the positive mK-dV solitons with $\mu_{e}$ and $\mu_{n}$ for $u_{0}=0.1, \alpha=5$ and $k=10$.

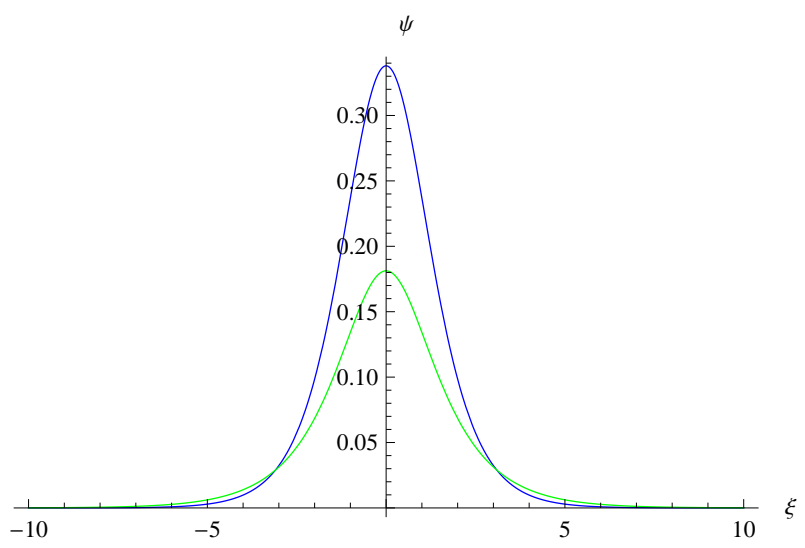

Figure 13. The variation of amplitude of both positive K-dV solitons (upper curve) and positive mK-dV solitons (lower curve) for $u_{0}=0.1, \mu_{n}=0.3, \mu_{e}=0.8, \alpha=10$ and $k=20$. 


$$
B=\frac{v_{p}^{3}}{2}
$$

Equation (22) is a mK-dV equation, exhibiting a stronger nonlinearity, smaller width, and larger propagation velocity of the nonlinear wave.

\subsection{Solution of mK-dV Equation}

The solution of Eq. (22) can be obtained by following the same procedure as K-dV equation. Therefore, the stationary solitary wave solution of $\mathrm{mK}-\mathrm{dV}$ equation can be witten as:

$$
\psi=\psi_{m} \operatorname{sech}\left(\zeta / \delta_{2}\right)
$$

where the amplitude $\psi_{m}$ and the width $\delta_{2}$ are given by $\psi_{m}=\sqrt{6 u_{0} / \beta B}$ and $\delta_{2}=\sqrt{u_{0} / B}$. The amplitude and width variation of $\mathrm{mK}-\mathrm{dV}$ solitons are nearly valid around critical value. The $\mathrm{mK}-\mathrm{dV}$ equation has a solitary wave solution around $\mu_{e}=\mu_{c}$. The formation of positive mK-dV solitons is shown in figure 8. This figure indicates that the amplitude of this wave increases with increasing the electron number density. Figures 9 and 10 show how the amplitude of this wave varies with some dusty plasma parameters. On the other hand, figures 11 and 12 show how the width of this wave varies with those dusty plasma parameters. The amplitude of this wave equation decreases with increasing the values of both $\alpha, \mu_{e}, \mu_{n}$ and amplitude increases with $k$. Reverse effects are found in the case of width of this wave which are shown in figures 11 and 12. Comparison of the solitary waves potential (amplitude) of K-dV and mK-dV solitons is shown in figure 13. From this figure it has been found that the potential of K-dV solitons is larger than the potential of mK-dV solitons with same values of dusty plasma parameters.

\section{Shock Waves}

\subsection{Derivation of Burgers Equation}

Now we derive the Burgers equation (BE). The DIA BE has been introduced by the following stretched coordinates $[34,44]$

$$
\left.\begin{array}{l}
\xi=\epsilon\left(x-v_{p} t\right) \\
\tau=\epsilon^{2} t
\end{array}\right\}
$$

where $v_{p}$ is the phase speed of DIA shock waves. By using Eqs. (5) and (26) in Eqs. (1)-(3), the same values of $u_{p}^{(1)}, n_{p}^{(1)}$, and $v_{p}$ are found as like as that of the K-dV equation. To the next higher order of $\epsilon$, we obtain a set of equations which, after using Eqs. (6) and (7), can be simplified as

$$
\begin{aligned}
& \frac{\partial n_{p}^{(1)}}{\partial \tau}-v_{p} \frac{\partial n_{p}^{(2)}}{\partial \xi}+\frac{\partial u_{p}^{(2)}}{\partial \xi}+\frac{\partial}{\partial \xi}\left(n_{p}^{(1)} u_{p}^{(1)}\right)=0 \\
& \frac{\partial u_{p}^{(1)}}{\partial \tau}-v_{p} \frac{\partial u_{p}^{(2)}}{\partial \xi}+u_{p}^{(1)} \frac{\partial u_{p}^{(1)}}{\partial \xi}+\frac{\partial \phi^{(2)}}{\partial \xi}-\eta \frac{\partial^{2} u_{p}^{(1)}}{\partial \xi^{2}}=0 \\
& \frac{\partial n_{p}^{(2)}}{\partial \xi}-\left(\frac{\mu_{e} \kappa_{3} \kappa_{4}}{\kappa_{2}^{2}}+\alpha^{2} \mu_{n}\right) \psi \frac{\partial \psi}{\partial \xi}-\frac{1}{v_{p}^{2}} \frac{\partial \phi^{(2)}}{\partial \xi}=0
\end{aligned}
$$

Now, combining Eqs. (27)-(29), we obtain a equation of the form:

$$
\frac{\partial \psi}{\partial \tau}+A \psi \frac{\partial \psi}{\partial \xi}=C \frac{\partial^{2} \psi}{\partial \xi^{2}}
$$

where the value of $\mathrm{A}$ is same as we obtained for $\mathrm{K}-\mathrm{dV}$ solitons and $\mathrm{C}$ is given by

$$
C=\frac{\eta}{2}
$$

Equation (30) is a Burger equation (BE). 


\subsection{Solution of Burgers Equation}

The stationary solution of this BE is obtained by transforming the independent variables $\zeta$ and $\tau$ to $\zeta=\xi-u_{0}$ and $\tau=\tau$, where $u_{0}$ is a constant velocity normalized by $C_{p}$, and imposing the appropriate boundary conditions, viz., $\psi \rightarrow 0, \partial \psi / \partial \zeta \rightarrow 0$, at $\zeta \rightarrow+\infty$. Thus, one can express the stationary solution of the BE as

$$
\psi=\psi_{m}[1-\tanh (\zeta / \delta)]
$$

where $\delta=2 C / u_{0}$ is the width and $\psi_{m}=u_{0} / A$ is the amplitude of the shock wave. It is clear from Eq. (12), for $A>0$ the dusty plasma supports compressive DIA shock waves which are associated with positive potential and for $A<0$ the dusty plasma supports rarefactive DIA shock waves which are associated with negative potential. On the other hand no shock waves exist for $A=0$. It is also noted that the nonlinear coefficient $\mathrm{A}$ is a function of $\alpha, k, \mu_{n}$ and $\mu_{e}$. Hence, in order to find the parametric regimes corresponding to $A=0$, we have to express one (viz. $\mu_{e}$ ) of these four parameters in terms of other parameters (viz. $\alpha$, $k$ and $\left.\mu_{n}\right)$. Therefore, $A\left(\mu_{e}=\mu_{c}\right)=0$ and the critical condition $\left(\mu_{c}\right)$ can be written as

$$
\mu_{c e}=\frac{1}{6 Q_{1}}\left[Q_{2}+Q_{3} \sqrt{Q_{4}+Q_{5}+Q_{6}}\right]
$$

where, $Q_{1}=1-4 k(1-k), Q_{2}=-1+4 k^{2}\left(1-6 \alpha \mu_{n}\right)-6 \alpha \mu_{n}(3+8 k), Q_{3}=1-2 k, Q_{4}=1+4 k\left(1+\alpha \mu_{n}\right)$, $Q_{5}=4 k^{2}\left(1-12 \alpha \mu_{n}\right)$ and $Q_{6}=4 \alpha \mu_{n}(9+k+27 \alpha-36 \alpha k) \cdot \mu_{c}$ is the critical value of $\mu_{e}$ above (below) which the shock waves with a positive (negative) potential can exist. We can find $A=0$ for a certain (critical) value of $\mu_{e}$, i.e. $A=0$ for $\mu_{e}=\mu_{c} \simeq 0.456$ for a set of dusty plasma parameters viz. $\mu_{n}=0.1$, $\alpha=10$ and $k=2$ in our present dusty plasma system. It is clear that the potential of shock wave becomes infinity at $\mu_{e}=\mu_{c}$ and the BE that we have derived is no loner valid at this condition, so shock waves are found only for $\mu_{e} \neq \mu_{c}$. Figure 14 shows the positive and negative potential shock structures for some dusty plasma parameters. This figure indicates that the amplitude of positive (negative) potential shock structures decreases (increases) with the increase of $\kappa$. Figure 15 shows the variation of potential $\left(\psi_{m}\right)$ of DIA shock waves with $\alpha$ and $\kappa$. It is found from this figure that the dusty plasma parameter $\alpha$ has a critical value. Above this value of $\alpha$ the shock structures associated with negative potential and below this value it associated with positive potential. Figure 16 represents the variation of width of the shock structures with positive kinematic viscosity $\eta$ for different values of positive ion fluid speed $u_{0}$. The width of the shock structures increases (decreases) with the increase of $\eta\left(u_{0}\right)$.

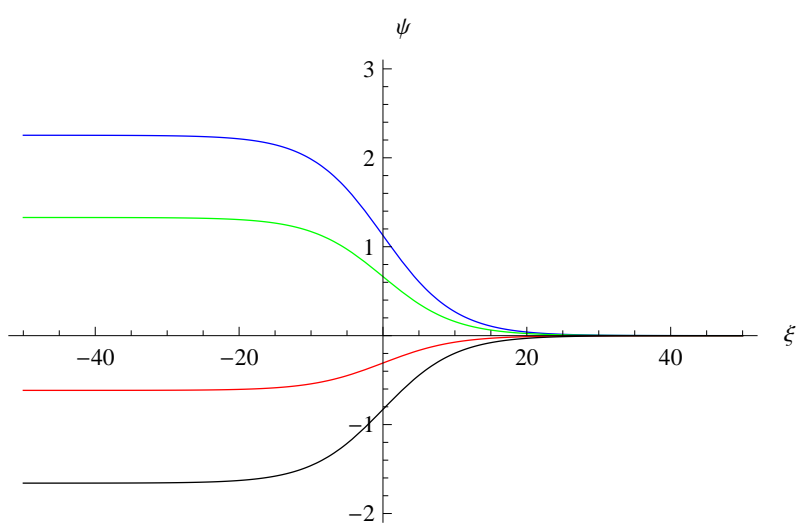

Figure 14. The variation of the positive and negative potential shock structures with $k$ for $u_{0}=0.1, \mu_{n}=0.1$, $\mu_{e}=0.5, \eta=0.5$ and $\alpha=1.4$. The blue curve for $k=2$, the green curve for $k=2.1$, the red curve for $k=1.7$ and the black curve for $k=1.8$. 


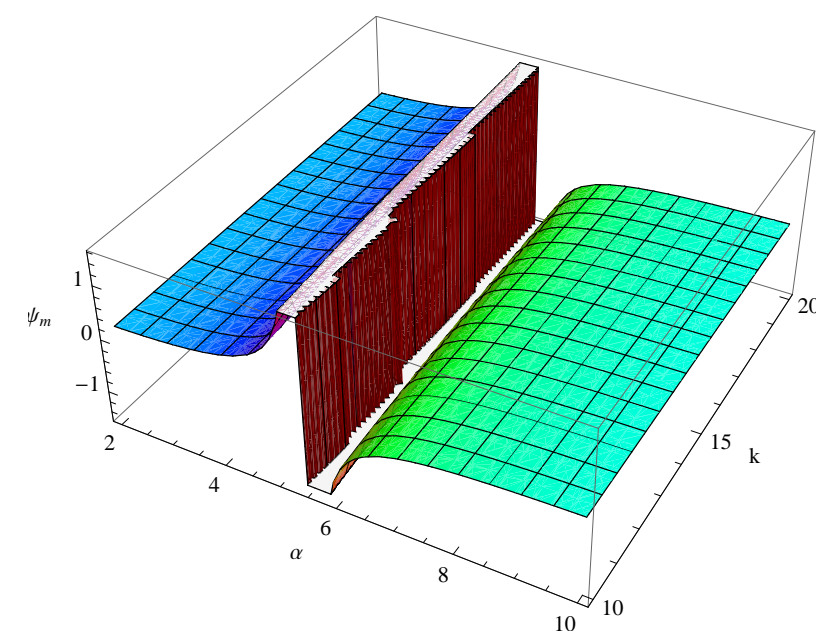

Figure 15. The variation of amplitude of the shock wave structures with $\alpha$ and $k$ for $u_{0}=0.1, \mu_{n}=0.1$ and $\mu_{e}=0.5$.

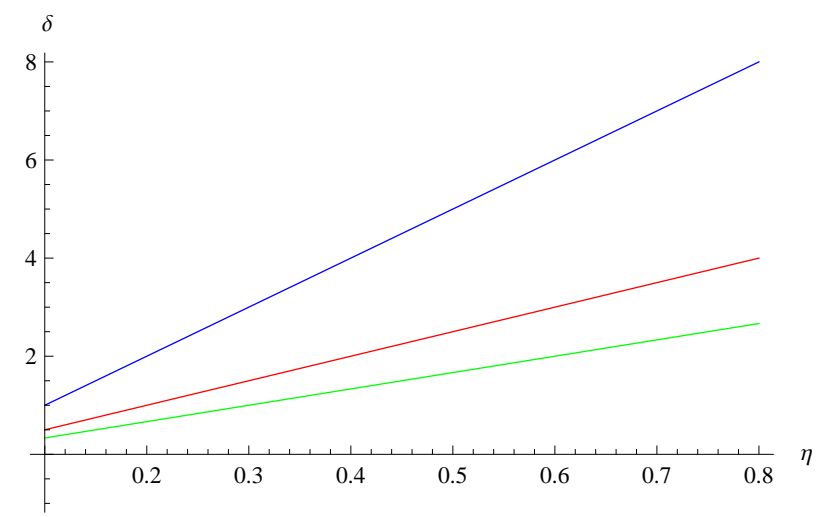

Figure 16. The variation of width of the shock wave structures with $\eta$. The blue curve for $u_{0}=0.1$, the red curve for $u_{0}=0.2$ and the green curve for $u_{0}=0.3$. 


\section{Discussion}

We have considered an unmagnetized dusty plasma system consisting of inertial cold positive ions, superthermal (Kappa distributed) electrons, Maxwellian distributed negative ions and positively charged stationary dust particles, and studied DIA solitary and shock waves. We have investigated the basic features (amplitude, width, phase speed, etc.) of these nonlinear waves (solitary and shock waves) associated with the considered dusty plasma system by deriving $\mathrm{K}-\mathrm{dV}, \mathrm{mK}-\mathrm{dV}$ equations and $\mathrm{BE}$ using the reductive perturbation method. The dusty plasma system under consideration supports finite amplitude solitary and shock waves, whose basic features strongly depend on the plasma parameters viz. $\alpha, k, \mu_{n}$ and $\mu_{e}$. The important finding that we obtained from our investigation can be summarized as follows:

1. We have found that $\mathrm{K}-\mathrm{dV}$ equation supports both compressive (positive) and rarefactive (negative) solitary waves, while $\mathrm{mK}-\mathrm{dV}$ equation supports only compressive solitary waves for above or below the critical value (i.e. when $\mu_{e}>\mu_{c}$ or $\mu_{e}<\mu_{c}$ ). The critical value of $\mu_{e}$ is $\simeq 0.456$.

2. The phase velocity of DIA waves decreases with increasing the number density of electron and the temperature ratio of electron and negative ion. That means, the phase velocity of nonlinear propagation becomes slower with increasing the number of electrons as well as the temperature in this plasma model.

3. The critical value of $\mu_{e}$ increases with increasing the ratio of electron and negative ion temperature and also with the spectral index which is shown in figure 2.

4. It has been found that solitary waves associated with both positive and negative potential due to the presence of kappa distributed superthermal electron.

5. The amplitude of the positive K-dV soliton increases up to certain of $\alpha$ (i.e., ratio of electron and ion temperature) and then it becomes infinity. On the other hand the amplitude of negative K-dV soliton decreases from infinity with increasing the value of $\alpha$. The amplitude of the negative K-dV soliton becomes larger when increasing the spectral index $k$ as well as the number density of negative ion $\left(\mu_{n}\right)$, which is shown in figure 5 .

6. The width of the K-dV soliton becomes smaller with increasing the values of $\alpha, \mu_{n}$ and $\mu_{e}$ which is shown in figure 6 and 7 .

7. The existence of only positive $\mathrm{mK}-\mathrm{dV}$ solitons is shown in figure 8 . The amplitude of this soliton becomes smaller with increasing the number density of electron $\left(\mu_{e}\right)$, the number density of negative ions $\left(\mu_{n}\right)$ and the ratio of electron and ion temperature $(\alpha)$ but it slightly increases with spectral index $(k)$ which are shown in figures 8-10.

8. The reverse results are obtained in the case of width of this soliton in our present plasma model which are shown in figures 11-12.

9. It is also clear from our investigation that the $\mathrm{mK}-\mathrm{dV}$ equation is a higher order nonlinear equation than our usual K-dV equation (depicted in figure 13).

10. The spectral index $k$ has a great contribution on the forming of solitary as well as shock profiles. For small values of $k$ the superthermal electrons in the tail of velocity distribution function increases and, vice versa. The superthermality effects on DIA shock profile are presented in figures 14 and 15 .

11. The amplitude of the positive shock profile gradually increases with $\alpha$, and it becomes infinity when $\alpha \simeq 4.8$. On the other hand, the magnitude of amplitude of the negative shock profile start to decreases from $\alpha \simeq 6$.

12. From this investigation, it is found that the width of the shock profile increases with the increase of $\eta$. In addition, it can also be said that with the increase of dissipation, the shock waves become smoother and weaker which is shown in figure 16.

In conclusion, the results which we obtained in our present investigation reveal that the relative temperature ratio, presence of positive as well as negative ions, presence of stationary dust particles and superthermality effect of electron play an important role in changing the phase speed, amplitude and width of the usual solitary and shock waves. We hope that the study of this nonlinear propagation of DIA solitary and shock waves should be useful in understanding the basic properties of the electrostatic disturbances in space and laboratory dusty plasmas where inertial cold positive ions, superthermal (kappa distributed) electrons, Maxwellian distributed negative ions and positively charged static dust are the major plasma species. 


\section{References}

1. M.C. Bagelmaan, R.D. Blanford and M.J. Rees, "Theory of extragalactic radio sources" Reviews of Modern Physics, vol. 56, pp. 255-351, 1984.

2. H.R. Miller and P. J. Witta, "Lecture Notes in Physics", Active Galactic Nuclei, vol. 307, 1988.

3. M. Tribeche, K. Aoutou, S. Younsi and R. Amour, "Nonlinear positron acoustic solitary waves", Physics of Plasmas, vol. 16, pp. 072103-5, 2009.

4. M. M. Masud, M. Asaduzzaman and A. A. Mamun, "Dust-ion-acoustic shock waves in a two-electrontemperature dusty plasma", Journal of Plasma Physics, vol. 79, pp. 215-219, 2012.

5. P.K. Shukla and V. P. Silin, "Dust ion-acoustic wave", Physical Scripta, vol. 45, pp. 508, 1992.

6. A. Barkan, N. D'Angelo and R. L. Merlino, "Experiment on ion-acoustic waves in dusty plasmas", Planetary and Space Science, vol. 44, pp. 239-242, 1996.

7. Y. Nakamura, H. Bailung and P. K. Shukla, "Observation of Ion-Acoustic Shocks in a Dusty Plasma", Physical Review Letters, vol. 83, pp. 1602-1605, 1999.

8. P. K. Shukla and A. A. Mamun, "Introduction to Dusty Plasma Physics", IoP, Bristol, UK, 2002.

9. P. K. Shukla and M. Rosenberg, "Boundary effects on dust-ion-acoustic and dust-acoustic waves in collisional dusty plasmas", Physics of Plasmas, vol. 6, pp. 1038-1040, 1999.

10. R. Bharuthrum and P. K. Shukla, "Large amplitude ion-acoustic solitons in a dusty plasma", Planetery and Space Science, vol. 40, pp. 973-977, 1992.

11. Y. Nakamura and A. Sharma, "Observation of ion-acoustic solitary waves in a dusty plasma", Physics of Plasmas, vol. 8, pp. 3921-3926, 2001.

12. P. K. Shukla and A. A. Mamun, "Solitons, shocks and vortices in dusty plasmas", New Journal of Physics, vol. 5, pp. 17.1-17.37, 2003.

13. S. K. El-Labany and E. F. El-Shamy, "Obliquely propagating electrostatistic solitary structures in a hot magnetized dusty plasma with vortex-like electron distribution", Astrophysics and Space Science, vol. 293, pp. 295-306, 2004.

14. A. A. Mamun and P. K. Shukla, "Dust-acoustic shock waves due to strong correlation among arbitrarily charged dust", Physics Letter A, vol. 373, pp. 3161-3164, 2009.

15. A. A. Mamun and R. A. Cairns, "Dust-acoustic shock waves due to strong correlation among arbitrarily charged dust", Physical Review E, vol. 79, pp. 055401-4, 2009.

16. M. G. M. Anowar, M. S. Rahman and A. A. Mamun, "Nonlinear dust-acoustic waves in a strongly coupled dusty plasma with vortexlike ion distribution", Physics of Plasms, vol. 16, pp. 053704-5, 2009.

17. O. Rahman and A. A. Mamun, "Dust-ion-acoustic solitary waves in dusty plasma with arbitrarily charged dust and vortex-like electron distribution", Physics of Plasmas, vol. 18, pp. 083703-5, 2011.

18. N. R. Kundu and A. A. Mamun, "Dust-ion-acoustic solitary waves in a dusty plasma with arbitrarily charged dust and non-thermal electrons", Journal of Plasma Physics, vol. 78, pp. 677-681, 2012.

19. V. M. Vasyliunas, "A survey of low-energy electrons in the evening sector of the magnetosphere with OGO 1 and OGO 3", Journal of Geophysical Research, vol. 73, pp. 2839-2884, 1968.

20. M. P. Leubner, "On Jupiter's whistler emission", Jouranal of Geophysical Researc: Space Science, vol. 87, pp. 6335-6338, 1982.

21. Z. Emami and H. R. Pakzad, "Solitons of KdV and modified KdV in dusty plasmas with superthermal ions ", Indian Journal of Physics, vol. 85, pp. 1643-1652, 2011.

22. S. Hussain, S. A. Shan, N. Akhtar and M. M. Masud, "Investigation of the properties of electrostatic IA solitary wave structures in negative ion magneto-plasmas with superthermal electrons", Astrophysics and Space Science, vol. 352, pp. 605-612, 2014.

23. E. Saberian, A. E. Kalejahi and M. A. Ghazi, "Large Amplitude Dust-acoustic Solitons in a Plasma Consisting of Superthermal Electron-positron Pairs and Dust Particulates", The Open Plasma Physics Journal, vol. 8, pp. 8-22, 2015.

24. O. R. Rufai, R. Bharuthram, S. V. Singh and G. S. Lakhina, "Effect of excess superthermal hot electrons on finite amplitude ion-acoustic solitons and supersolitons in a magnetized auroral plasma", Physics of Plasmas, vol. 22, pp. 102305-7, 2015.

25. K. Arshad, A. Rahman, and S. Mahmood, "Landau damping of Langmuir twisted waves with kappa distributed electrons", Physics of Plasmas, vol. 22, pp. 112114-7, 2015.

26. S. Mayout, B. Sahu and M. Tribeche, "Time evolution of nonplanar dust ion-acoustic solitary waves in a charge varying dusty plasma with superthermal electrons", Physics of Plasmas, vol. 22, pp. 123703-6, 2015.

27. D. Summers and R. M. Thorne, "The modified plasma dispersion function", Physics Fluids B, vol. 3, pp. 1835-1847, 1991.

28. R. L. Mace and M. A. Helberg, "A dispersion function for plasmas containing superthermal particles", Physics of Plasmas, vol. 2, pp. 2098-2109, 1995. 
29. T. K. Baluku and M. A. Hellberg, " Dust acoustic solitons in plasmas with kappa-distributed electrons and/or ions", Physics of Plasmas, vol. 15, pp. 123705-11, 2008.

30. M. A. Hellberg, R. L. Mace, T. K. Baluku, I. Kourakis and N. S. Saini, " Comment on ŞMathematical and physical aspects of Kappa velocity distribution $\breve{T}$ [Phys. Plasmas14, 110702 (2007)]", Physics of Plasmas, vol. 16, pp. 094701-5, 2009.

31. H. Washimi and T. Taniuti, "Propagation of ion-acoustic solitary waves of small amplitude", Physics Review Letters, vol. 17, pp. 996-997, 1966.

32. V. I. Karpman, "Nonlinear Waves in Dispersive Media", Pergamon, Oxford, UK, 1975.

33. T. K. Baluku, M. A. Hellberg, I. Kourakis and N. S. Saini, " Dust ion acoustic solitons in a plasma with kappa-distributed electrons", Physics of Plasmas, vol. 17, pp. 053702-11, 2010.

34. M. S. Alam, M. M. Masud and A. A. Mamun, "Effects of bi-kappa distributed electrons on dust-ion-acoustic shock waves in dusty superthermal plasmas", Chinese Physics B, vol. 22, pp. 115202-6, 2013.

35. M. G. Shah, M. M. Rahman, M. R. Hossen and A. A. Mamun, "Roles of Superthermal Electrons and Adiabatic Heavy Ions on Heavy-Ion-Acoustic Solitary and Shock Waves in a Multi-Component Plasma", Communications in Theoretical Physics, vol. 64, pp. 208-214, 2015.

36. H. Schamel, "Stationary solitary, snoidal and sinusoidal ion acoustic waves", Plasma Physics, vol. 14, pp. 905-924, 1972.

37. H. Schamel, "A modified Korteweg-de Vries equation for ion acoustic waves due to resonant electrons", Journal of Plasma Physics, vol. 9, pp. 377-387, 1973.

38. H. Schamel, "Analytic BGK modes and their modulational instability", Journal of Plasma Physics, vol. 13, pp. 139-145, 1975.

39. P.K. Shukla and M.Y. Yu, "Exact solitary ion acoustic waves in a magnetoplasma", Journal of Mathematical Physics, vol. 19, pp. 2506-2508, 1978.

40. L.C. Lee and J.R. Kan, "Nonlinear ion-acoustic waves and solitons in a magnetized plasma", Physics of Fluids, vol. 24, pp. 430-433, 1981.

41. P.K. Shukla, M.Y. Yu and R. Bharuthram, "Linear and nonlinear dust drift waves ", Journal of Geophysical Research: Space science, vol. 96, pp. 21.343-21.346, 1991.

42. A. Mannan and A. A. Mamun, "Nonplanar dust-acoustic Gardner solitons in a four-component dusty plasma", Physical Review E, vol. 84, pp. 026408-6, 2011.

43. N. C. Lee, "Small amplitude electron-acoustic double layers and solitons in fully relativistic plasmas of two-temperature electrons", Physics of Plasmas, vol. 16, pp. 042316-10, 2009.

44. M. M. Masud, S. Sultana and A. A. Mamun, "Effects of double temperature superthermal electrons on dust-ion-acoustic shock waves in electron-positron-ion dusty plasmas", Astrophysics and Space Science, vol. 348, pp. 99-105, 2013. 